\title{
Stability of the Sun-Earth-Moon system
}

\author{
V. Szebehely and R. McKenzie \\ The University of Texas, Austin \\ (Received 21 December 1976)
}

\begin{abstract}
The models of the restricted and general problems of three bodies are used to determime the stability of the Sun-Earth-Moon system by means of surfaces of zero velocity. Hill's result is verified by the model of the restricted problem as long as the ratio $m_{\mathrm{E}} / m_{\mathrm{S}} \geq 2.52 \times 10^{-6}$. The model of the general problem, on the other hand, contradicts this result and we show that the eccentricity of the Earth's orbit renders the system unstable by opening the surface of zero velocity. It may be concluded, therefore, that the Moon may escape from the Earth and may become a planet or, in reverse, that the planetary origin and the capture of the Moon by the Earth becomes a strong dynamic possibility.
\end{abstract}

\section{INTRODUCTION}

$\mathrm{T}$ HE existence of zero-velocity surfaces in the general problem of three bodies was shown by Golubev (1968). Similar results were obtained by Smale (1970), Saari (1975), Marchal (1975), Bozis (1976), and Zare (1976), who all studied various aspects of the topology of the manifold of motion. In a series of papers we applied the above results to investigate possible regions of motion of classical triple stellar configurations (Szebehely and Zare 1977), to study the long-time behavior of the solar system (Szebehely and McKenzie 1977) and to show the use of bifurcation theory in the stability of the Sun-Jupiter-Saturn system. The present paper treats the behavior of the Sun-Earth-Moon system by means of models using zero-velocity surfaces.

The idea to apply zero-velocity surfaces to investigate the stability of the lunar system was originated by Hill (1878), who showed that the Moon's orbit is inside a zero-velocity oval, which in turn is inside the limiting critical figure-eight curve of zero velocity. In this way, using Hill's simplified model or the model of the restricted problem of three bodies one may show that neither can the Moon leave the Earth, nor can it be captured. The model of the restricted problem neglects the eccentricity of the Earth's orbit and the effect of the Moon's mass. For this reason it is desirable to refine the dynamic model used so that the above two effects may be included in the theory. This is made possible by the use of the model of the general problem of three bodies as suggested to us by R. Vicente (1975). In the following the relation between zero-velocity curves and stability is first shortly reviewed, then a quantitative measure of stability is introduced, and finally some numerical results are given regarding the title problem.

\section{DEFINITION OF STABILITY}

Stability is defined in this paper along the lines proposed by Hill (1878). If the orbit is enclosed by a zero- velocity surface, the corresponding body may not penetrate this surface and the motion can not change its basic characteristics. As long as the type of motion is unchanged we call it stable. For instance, in the restricted problem of three bodies when the orbit of the body with zero mass is inside a zero-velocity oval drawn around one of the primaries, i.e., when its Jacobian constant $C_{\mathrm{ac}}$ is larger than the critical value of the Jacobian constant $C_{\mathrm{cr}}$ (corresponding to the libration point located between the primaries, $L_{2}$ ), we speak about stability. The body with zero mass may collide with one of the primaries but it may not leave the inside of its zero-velocity curve when $C_{\mathrm{ac}} \geq C_{\mathrm{cr}}$. If this inequality is reversed the zero-velocity curve corresponding to the body with zero mass opens up at $L_{2}$ and the body may switch its motion from around one primary to the other and the character and type of the motion may change. In this paper we associate the loss of stability with such basic change of motion in the general as well as in the restricted problem. The corresponding measures of stability, therefore, are

$$
S=\frac{C_{\mathrm{ac}}-C_{\mathrm{cr}}}{C_{\mathrm{cr}}}, \quad S=\frac{s_{\mathrm{ac}}-s_{\mathrm{cr}}}{s_{\mathrm{cr}}}
$$

for the restricted and for the general problem, respectively. Here $C$ is the Jacobian constant in the restricted problem and $\left.s=-\left(c^{2} H\right) / G^{2} \bar{m}^{5}\right)$ is the stability parameter in the general problem. The Jacobian constant is defined as $C=2 \Omega-v^{2}$, where

$$
\Omega=\frac{1}{2}\left[(1-\mu) r_{1}^{2}+\mu r_{2}^{2}\right]+\frac{1-\mu}{r_{1}}+\frac{\mu}{r_{2}},
$$

$\mu$ is the mass parameter given in terms of the primary masses by $\mu=m_{2} /\left(m_{1}+m_{2}\right)$, and $m_{2} \leq m_{1}$. Here $r_{1}$ and $r_{2}$ are the distances between the primaries and the third ("infinitesimal") body, whose velocity in the synodic coordinate system is $v$. For the general problem $c$ and $H$ represent the total angular momentum and the (negative) total energy of the system, $G$ is the gravitational constant, and $\bar{m}$ is the average mass. For the computation of the critical and actual values of $C$ and $s$ we refer the reader to our previous publications (Szebehely 1967; 
Szebehely and Zare 1977; Szebehely and McKenzie 1977).

The relation of our definition of stability to bifurcation theory is well known. At the critical or bifurcation value ( of $C$ or $s$ ) the character of the motion may change and therefore, stability is defined by $S>0$.

It must be noted that at bifurcation a different behavior does not necessarily occur, but it may occur. Furthermore, limiting ranges of motion may be defined with inequalities of various sharpnesses obtained by different estimates. For instance Sundman's inequality forms the basis of the estimates used in this paper to establish the limiting ranges of motion. There exist, however, different forms of this inequality with different sharpnesses and their use may furnish different stability criteria. This formulation of the problem was proposed by Broucke (1976).

Finally, we add that if $S$ is a large positive number the actual system is far removed from its critical condition and we call the system "more stable" than when $S$ is a smaller positive number. In this latter case uncertainties in our knowledge regarding the physical description of the system or effects not included in our theory may result in $S<0$, corresponding to an open zero-velocity surface and to instability, or rather to the possibility of instability, when the Moon may leave the Earth or it may be captured. A highly unstable system has a large negative value for $S$ indicating that the system is far removed from stability. (Note that $s_{\mathrm{cr}} \cdot C_{\mathrm{cr}} \neq 0$ as long as at least two of the three participating masses are not zero.)

\section{NUMERICAL RESULTS}

\section{A. The Model of the Restricted Problem}

Hill's (1878) result and statement regarding the stability of the lunar orbit is well known. If the model of the restricted problem is used as an improvement over (what is known today as) Hill's problem we still have stability. The Jacobian constant of the Moon is $C_{\mathrm{ac}}=3.0012$ while the critical value is $C_{\mathrm{cr}}=3.0009$. The measure of stability is $S=10^{-4}>0$, therefore, the system is stable. It must be recalled, however, that this model neglects the eccentricity of the Earth's orbit and the effect of the Moon's mass.

It is of interest to study the effect of the mass parameter on the stability of the system. The usual definition of the mass parameter is

$$
\mu=\frac{m_{\mathrm{E}}+m_{\mathrm{M}}}{m_{\mathrm{S}}+m_{\mathrm{E}}+m_{\mathrm{M}}} .
$$

If the Earth's and Moon's masses are changed by a factor $\gamma=m_{\mathrm{E}}^{\prime} / m_{\mathrm{E}}=m_{\mathrm{M}}^{\prime} / m_{\mathrm{M}}$ (where $m_{\mathrm{E}}, m_{\mathrm{M}}$ are the original and $m_{\mathrm{E}}^{\prime}, m_{\mathrm{M}}^{\prime}$ the new masses) we have for the new value of the mass parameter

$$
\mu^{\prime}=\frac{\gamma\left(m_{\mathrm{E}}+m_{\mathrm{M}}\right)}{m_{\mathrm{S}}+\gamma\left(m_{\mathrm{E}}+m_{\mathrm{M}}\right)} .
$$

The actual and critical values of the Jacobian constant and therefore the measure of stability depend now on $\mu^{\prime}$ (or on $\gamma$ ). The results of such calculations are that as long as $\gamma \geq 0.83$ the system preserves its stability. This means that if the present value of the mass parameter $\mu=$ $3.0404 \times 10^{-6}$ is reduced to less than $\mu^{\prime}=2.52 \times 10^{-6}$ the system becomes unstable. The physical interpretation of this result may be that as the Sun's mass is increased and the Earth's mass is decreased the Earth is not offering a sufficiently high potential to restrain the motion of the Moon.

The above computations assumed that the Moon's orbit and its mean motion did not change while the masses were varied. If we allow for such changes the critical value of $\gamma$ becomes 0.4 , that is as long as the mass parameter is larger than $1.22 \times 10^{-6}$ the system is stable.

\section{B. The Model of the General Problem of Three Bodies}

For the purposes of computing the actual and critical values of the stability parameter, the following astronomical constants are used:

$$
\begin{aligned}
m_{\mathrm{M}} / m_{\mathrm{E}} & =0.0123 ; \\
m_{\mathrm{S}} / m_{\mathrm{E}} & =332946.0 ; \\
a_{\mathrm{E}} / a_{\mathrm{M}} & =389.1845 ; \\
e_{\mathrm{M}} & =0.0549 ; \\
e_{\mathrm{E}} & =0.01675 .
\end{aligned}
$$

Here $a_{\mathrm{E}}, e_{\mathrm{E}}, a_{\mathrm{M}}$, and $e_{\mathrm{M}}$ are the semimajor axes and eccentricities of the Earth's and Moon's orbits.

The Keplerian total energy of the system is

$$
H=-\frac{G m_{\mathrm{E}} m_{\mathrm{S}}}{2 a_{\mathrm{E}}}\left[1+\frac{m_{\mathrm{M}}}{m_{\mathrm{E}}}\left(1+\frac{m_{\mathrm{E}}}{m_{\mathrm{S}}} \frac{a_{\mathrm{E}}}{a_{\mathrm{M}}}\right)\right] .
$$

The angular momentum (assuming coplanar motion) is

$$
\begin{aligned}
c=\frac{G m_{\mathrm{E}} m_{\mathrm{S}}}{\sqrt{M G}} \sqrt{a_{\mathrm{E}}}\left\{\left(1+\frac{m_{\mathrm{M}}}{m_{\mathrm{E}}}\right) \sqrt{1-e_{\mathrm{E}}^{2}}\right. \\
+\frac{m_{\mathrm{M}}}{m_{\mathrm{S}}}\left[\frac{a_{\mathrm{M}}}{a_{\mathrm{E}}}\left(1-e_{\mathrm{M}}^{2}\right)\right]^{1 / 2} \\
\left.\times\left(\frac{M}{m_{\mathrm{E}}+m_{\mathrm{M}}}\right)^{1 / 2}\right\},
\end{aligned}
$$

where $M=m_{\mathrm{S}}+m_{\mathrm{E}}+m_{\mathrm{M}}$.

Denoting the quantities in the brackets in Eqs. (5) and (6) by $\phi$ and $\psi$, we have

$$
s=-\frac{H c^{2}}{\bar{m}^{5} G^{2}}=-\frac{3^{5}}{2}\left(\frac{m_{\mathrm{E}}}{m_{\mathrm{S}}}\right) \frac{\phi \psi^{2}}{\sigma^{6}},
$$

where

$$
\sigma=1+\frac{m_{\mathrm{E}}}{m_{\mathrm{S}}}+\frac{m_{\mathrm{M}}}{m_{\mathrm{S}}}
$$

Note that the dimensionless factors $\phi, \psi$, and $\sigma$ contain 
only mass ratios, the ratios of the semimajor axes, and the eccentricities.

The critical value of $s$ depends only on the masses and may be computed by solving a fifth-order algebraic equation as shown for instance by Szebehely and Zare (1977). The measure of stability using the above astronomical constants becomes $S=-2.75 \times 10^{-4}$. Since it is negative, the zero-velocity surfaces are open and the Moon may escape the Earth or it may be captured by it.

The effects of the masses, semimajor axes, and eccentricities on the stability may be studied and the following results may be of some possible cosmological interest.

Changing the masses of the Moon and of the Earth (as before) by a factor of $\gamma$ results in stability of the system when $\gamma \geq 25.74$. This is not surprising since as $\gamma$ increases the system approaches the configuration similar to a classical triple stellar system. The "inner binary" consists of the Earth and the Moon while the "outer star" is the Sun. When $\gamma=50000$ the measure of stability becomes 0.8 , which is a good average value for a triple stars having an inner binary of $m_{\mathrm{E}}=0.15 m_{\mathrm{S}}$ and $m_{\mathrm{M}}=$ $0.0123 m_{\mathrm{E}}$ and an outer star of $m_{\mathrm{S}}$.

The decrease of the ratio $a_{\mathrm{M}} / a_{\mathrm{E}}$ also results in stability. Introducing the parameter $\delta$ (similarly to $\gamma$ ) as the ratio of the changed semimajor axis of the lunar orbit to its original value by $\delta=a_{\mathrm{M}}^{\prime} / a_{\mathrm{M}}$, we find that stability is associated with $\delta \leq 0.0488$. In other words, if the Earth-Moon distance were reduced to at least 18758 $\mathrm{km}$ the system would become stable. Tidal effects and the effects of gravitational harmonics not included in our theory will reduce the significance of this rather expected result.

The effect of the eccentricity of the orbit of the Earth is pronounced on the measure of stability of the system; in fact the reason for the discrepancy between the stable behavior found in the restricted problem and the instability established using the model of the general problem may be contributed to the eccentricity of the Earth's orbit. If the masses and semimajor axes of the Earth and of the Moon as well as the eccentricity of the lunar orbit are kept at their actual values and the Earth's orbital eccentricity $e_{\mathrm{E}}$ is changed we find stability as long as $e_{\mathrm{E}}$ $\leq 0.0023$, but instability above this value. The explanation is that the critical value of the stability parameter as mentioned before is independent of the eccentricities. The actual value of $s$, on the other hand, depends on $e_{\mathrm{E}}$ and $e_{\mathrm{M}}$ through $c$ or through $\psi$ as shown by Eq. (6). The functional form is

$$
\psi=1.0123 \sqrt{1-e_{\mathrm{E}}^{2}}+3.4 \times 10^{-6} \sqrt{1-e_{\mathrm{M}}^{2}},
$$

or

$$
\psi^{2} \cong 1.02475\left(1-e_{\mathrm{E}}^{2}\right)
$$

Consequently, $s_{\mathrm{ac}}\left(e_{\mathrm{E}}\right)=s_{\mathrm{ac}}(0)\left(1-e_{\mathrm{E}}^{2}\right)$, where $s_{\mathrm{ac}}(0)$ is the stability parameter of the system with the Earth on circular orbit. The critical value of the stability parameter is $s_{\mathrm{cr}}=3.414925 \times 10^{-15}$ and using the astronomical constants given before $s_{\mathrm{ac}}(0)=3.4149609 \times$ $10^{-15}$. Therefore, as long as $e_{\mathrm{E}} \leq\left\{1-\left[s_{\mathrm{cr}} / s_{\mathrm{ac}}(0)\right]\right\}^{1 / 2}$ $=0.0023$ the measure of stability is positive and the motion is stable.

We conclude the treatment of the title problem by comparing some values found previously regarding the measure of stability $(S)$ with the values given in this paper. The value of $S$ found for the Sun-Earth-Moon system, $-2.75 \times 10^{-4}$, does not represent a large instability when compared to known stable systems such as triple stellar systems for which $S$ is between 0.1 ( $\lambda$ Tau) and 4.3 (Algol). The simplified model of the solar system (Sun-Jupiter-Saturn) corresponds to $S=3.6 \times 10^{-2}$, consequently, it is "less stable" than triple stellar systems but definitely "more stable" than the "slightly unstable" Sun-Earth-Moon system.

The partial supports of the Bureau of Engineering Research, University of Texas at Austin, and of the Scientific Affairs Division of NATO are gratefully acknowledged. Conferences with our associates at the Center for Celestial Mechanics at the University of Texas especially with Dr. R. Broucke, Dr. R. Duncombe, Dr. W. Jefferys, and Dr. P. Nacozy were of considerable help in clarifying some of the aspects of this research. Discussions and extensive correspondence with Dr. C. Marchal were most beneficial.

\section{REFERENCES}

Bozis, G. (1976). Astrophys. Space Sci. 43, 355.

Broucke, R. (1976). Private communication.

Golubev, V. G. (1968). Doklady Akad. Nauk SSSR 180, 308 (translated Sov. Phys. 13, 373).

Hill, G. W. (1878). Am. J. Math. 1, 5, 129, 245.

Marchal, C. (1975). "Qualitative Methods and Results in Celestial Mechanics," ONERA Rep. No. 1975-77. Also in Long-time Predictions in Dynamics (Reidel, Holland).

Marchal, C., and Saari, D. (1975). Celest. Mech. 12, 115.

Smale, S. (1970). Invent. Math. 11, 45.

Szebehely, V. (1967). Theory of Orbits (Academic, New York)

Szebehely, V., and McKenzie, R. (1977). Astron. J. 82, 79.

Szebehely, V., and Zare, K. (1977). Astron. Astrophys. To be pub. lished.

Vicente, R. (1975). Private communication.

Zare, K. (1976). Doctoral dissertation, Univ. Texas.

V. Szebehely AND R. MCKenZIE: Department of Aerospace Engineering, University of Texas, Austin, Texas 78712 